\title{
Molecular and cellular pathology: summary
}

\author{
Michael N. Moore \\ Plymouth Marine Laboratory (NERC), Citadel Hill, Plymouth, Devon PL1 2PB, United Kingdom
}

Introduction. The aim of the molecular and cellular pathology group in the Bremerhaven Workshop was to test for evidence of biological changes which were indicative of exposure to toxic chemicals, as well as to their adverse effects. Much of the study focused on the liver of the flatfish dab Limanda limanda. The liver was chosen as it is an integrator of many functions including detoxication/activation of toxic chemicals, digestion and storage, excretion and synthesis of the egg yolk protein vitellogenin. There is also a considerable body of literature on pollutant chemical impact on the cellular pathology of fish liver to aid interpretation.

The approach was based on the detection of molecular and cellular changes resulting from chemical contamination. The identification of early onset changes, which may ultimately lead to overt disease is a key requirement in assessment of the effects of environmental pollution. Diseased fish are likely to be rapidly eliminated from the population, hence assessment based on such fish may prove difficult, and even if samples are available, higher level complications resulting from the primary lesion will be a confounding factor. In fact, it is only through the mechanistic linking of changes in molecular and cellular processes with the later pathological endpoints that it will be possible to establish causal relationships.

An additional component of the investigation examined developmental abnormalities and chromosomal aberrations in embryos of dab sampled along the transect.

An integrated perspective. The results of this investigation of dab in the North Sea show a considerable degree of internal consistency, as well as clearly following the trend of declining levels of chemical contaminants along the transect towards the central North Sea. A pattern emerges from the data showing lysosomal, cellular, tissue and embryonic damage of the type expected due to the toxic action of xenobiotics. The additional evidence of proliferation of the smooth endoplasmic reticulum in hepatocytes, together with an increase in the activity of the inducible detoxication/activation enzyme EROD, further supports the premise that the fish have been exposed to inducerxenobiotics, which is consistent with the analytical chemical data (Cofino et al. 1992). Proliferation of smooth endoplasmic reticulum (SER) and induction of EROD also provide a reasonable mechanistic explanation for the enhanced generation of superoxide radicals, through futile cycling, at the most contaminated sites, while radical attack on lysosomes is probably a contributory factor to the clear evidence for lysosomal membrane injury and consequent dysfunction. The presence of phospholipidosis (accumulation of phospholipid membrane components in lysosomes) in severely damaged livers is also consistent with autophagy of chemically damaged proliferated endoplasmic reticulum and failure of the functionally impaired lysosomes to degrade the ingested membranes, which results in the characteristic pathological presentation of this lysosomal storage disease condition.

The presence of foci of altered cells at the most contaminated sites needs to be interpreted carefully. Comparison with the data from other species lends support to the conclusion that the high prevalence of foci is indicative of exposure to xenobiotic pollutants. The relationship of foci to neoplastic change in dab cannot at the present state of our knowledge be determined and requires further experimental investigation. It is, perhaps, worth commenting that neoplastic change in itself is of little significance in ecological terms unless the incidence in the population is extremely high. The value of pursuing the "neoplastic pathway' is that this type of disease is probably indicative of exposure to carcinogenic xenobiotics, although 
whether this occurs in embryos and larvae associated with xenobiotic-rich surface microlayer or else as juveniles and adults in contact with bottom sediment and contaminated prey organisms is still an open question. The further finding that the prevalences of ras-oncoprotein positivity and foci of altered cells in livers of dab were similarly distributed would support the hypothesis that the initial steps in the process are occurring in the adult fish, as the involvement of ras-oncoprotein in the process of carcinogenesis is believed to occur at a very early stage (Moore \& Evans 1992).

A direct approach to the question of exposure to carrcinogens involved the use of a battery of assays for the

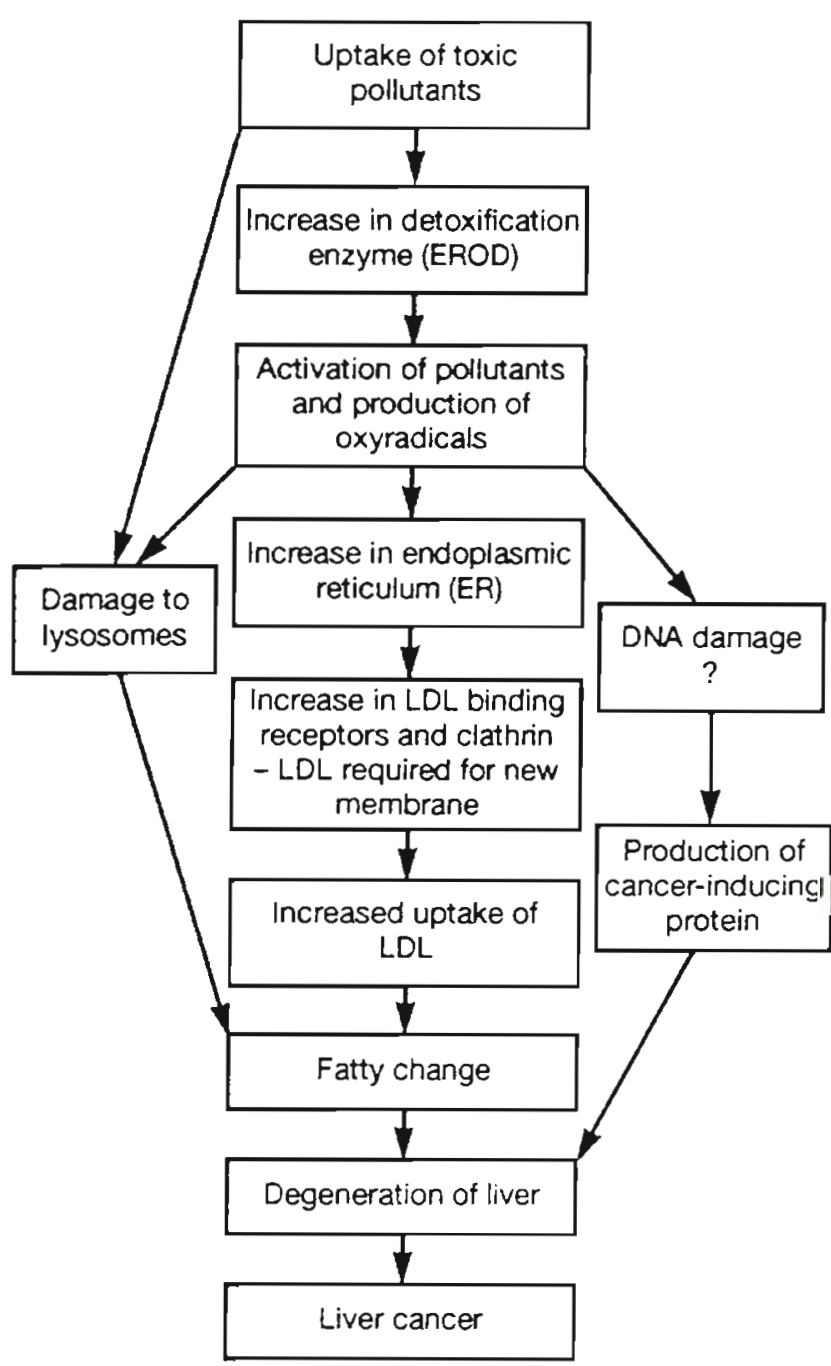

Fig. 1. Outline scheme of possible early events in the development of degenerative and neoplastic disease of fish liver. $\mathrm{LDL}=$ low density lipoprotein. Scheme is based on data from this workshop plus additional findings on ras-oncoprotein (cancer-inducing protein) from Moore \& Evans (1992) and Lowe (unpubl.) and Moore et al. (1991) on LDL, LDL receptors and clathrin detection of genotoxic damage and production of metabolites of chemical pro-mutagens. Liver samples were analysed for bulky hydrophobic DNA adducts and oxidised bases (8-hydroxydeoxyguanosine); no significant differences were seen between stations along the transect. Mutagenicity assays did not detect mutagens in liver extracts of deconjugated bile samples. There was no evidence of pollution-related unscheduled DNA synthesis in dab liver cells. Liver enzymes, however, activated 2-acetylaminofluorene to mutagenic derivatives to a significantly greater extent in fish from Stns 3 \& 5 than from Stns $6 \& 7$

Dab embryo abnormalities and chromosomal aberrations (Cameron \& Berg 1992) were highest at the inshore contaminated Stns $3 \& 5$, as well as Stn 9 on the eastern side of the Dogger Bank, and lowest at Stns $7 \&$ 8. Chromosomal aberrations were highest at Stns 3 \& 5, as compared to Stns $7 \& 8$, with an upward trend in frequency at Stn 9.

This integrated study probably represents the only one to date on such a large scale where a range of early cellular markers of xenobiotic-induced cell injury have been coupled with a detailed histopathological investigation of the status of the liver in flatfish. The value of the cellular pathological approach is clear from the unequivocal demonstration of liver damage at stations known to be contaminated, and that the nature of the liver injury is due to exposure to contaminant xenobiotics. It must be emphasised that early onset cellular markers should be used in an integrated manner and not in isolation, otherwise a distorted interpretation may be drawn from the data. If they are used appropriately, then it is possible to build up rapidly an holistic picture of the pattern of liver injury and to identify the probable causative factors, although not as specific single chemical entities. However, there is seldom any call for such specificity as most contaminated situations consist of numerous xenobiotics, many of which will exert their toxicities in concert. This argues very strongly for the use of cellular pathology as an integrated multi-parameter biomarker or linked network of biomarkers.

The endpoint in this type of approach is the use of cellular pathology as an integrated predictive biomarker of the health status of individuals in a population. It is not for the pathologist to extrapolate to the broader ecological consequences; this lies in the realms of environmental epidemiology and population ecology, and certainly this latter discipline appears to lack the necessary tools for extrapolation at present (Underwood \& Peterson 1988). It is considered that it is sufficient for the reasonable assessment of contaminant impact to demonstrate pathological change of a degree likely to either impair, or to be a predictor of impairment of, animal function and performance in a 
significant proportion of a representative sample taken from the population. This in itself is cause for environmental concern without any need to demonstrate ecological effects, which are probably impossible to interpret in terms of causality.

Conclusions. The findings clearly demonstrate degenerative changes in liver cells and embryos from the most contaminated inshore sites. The most obvious early onset cellular 'distress signal' was given by the various measurements of lysosomal damage (membrane stability, neutral red retention, binding of acridine orange, enlargement and toxic lipidasis). These alterations together with the higher-order lesions such as fatty change and preneoplastic foci are all biomarkers of liver degeneration in the 'non-overtly' diseased female dab used in this study. The increased endoplasmic reticulum, its associated EROD activity and activation of pro-mutagens, together with the increased production of oxyradicals can be considered as probable biomarkers of exposure to contaminant xenobiotics, as indicated by the liver PCB data.

The results indicate that future monitoring studies should include a suite of molecular and cell pathological techniques, which would provide a link between biochemical and histopathological results. This would provide a better understanding of the sequence of events between contaminant exposure and its probable effects, as is shown in Fig. 1, identifying new links to help environmental decision makers. A further benefit would be to aid in the assessment of recovery during environmental bioremediation. No single tech- nique provides an adequate index of pollutant effect, rather we advocate the use of a comprehensive suite of clinical-type tests from those now available to cellular and developmental pathologists. In fact, this study has clearly demonstrated that an integrated pathological approach can identify both exposure to xenobiotics and induced cell injury. In order to further strengthen this biomarker approach there is a distinct need to develop cellular markers that will demonstrate whether the degree of pathological change is either reversible or irreversible. Only in this way will we derive biomarkers which are truly prognostic as well as being diagnostic.

\section{LITERATURE CITED}

Cameron, P., Berg, J. (1992). Morphological and chromosomal aberrations during embryonic development in dab Limanda limanda. Mar. Ecol. Prog. Ser. 91. 163-169

Cofino, W P., Smedes, F., de Jong, S. A., Abarnou, A., Boon, J. P., Oostingh, I., Davies, I. M., Klungsøyr, J., Wilhelmsen, S., Law, R. I., Whinnett, J. A., Schmidt, D., Wilson, S. (1992). The chemistry programme. Mar. Ecol. Prog. Ser. 91: $47-56$

Moore, M. N., Evans, B. M. (1992). Immunocytochemical detection of ras oncopeptide in liver cells of flatfish (dab) from an historically contaminated site in the North Sea. Mar. environ. Res. 34: 33-38

Moore, M. N., Lowe, D. M., Bucke, D., Dixon, P. (1991). Molecular and cellular markers of pollutant exposure and Liver damage in fish. ICES, C.M. 1991/E: 23

Underwood, H. J., Peterson, C. H. (1988). Towards an ecological framework for investigating pollution. Mar. Ecol. Prog. Ser. 46: 227-234 